\title{
Whole-exome sequencing confirms implication of VPS13D as a potential cause of progressive spastic ataxia
}

\author{
Christelle M. Durand ${ }^{1,2^{*}}$ (D) Chloé Angelini ${ }^{2,3}$, Vincent Michaud ${ }^{1,3}$, Claire Delleci ${ }^{2,4,5}$, Isabelle Coupry ${ }^{1,2}$, \\ Cyril Goizet ${ }^{1,2,3}$ and Aurelien Trimouille $e^{1,6}$
}

\begin{abstract}
Background: VPS13D is a large ubiquitin-binding protein playing an essential role in mitophagy by regulating mitochondrial fission. Recently, VPS13D biallelic pathogenic variants have been reported in patients displaying variable neurological phenotypes, with an autosomic recessive inheritance.

The objectives of the study were to determine the genetic etiology of a patient with early onset sporadic progressive spastic ataxia, and to investigate the pathogenicity of VPS13D variants through functional studies on patient's skin fibroblasts.

Case presentation: We report the case of a 51-year-old patient with spastic ataxia, with an acute onset of the disease at age 7 . Walking difficulties slowly worsened over time, with the use of a wheelchair since age 26 . We have used trio-based whole-exome sequencing (WES) to identify genes associated with spastic ataxia. The impact of the identified variants on mitochondrial function was assessed in patient's fibroblasts by imaging mitochondrial network and measuring level of individual OXPHOS complex subunits. Compound heterozygous variants were identified in VPS13D: c.946C > T, p.Arg316* and c.12416C > T, p.(Ala4139Val). Primary fibroblasts obtained from this patient revealed an altered mitochondrial morphology, and a decrease in levels of proteins from complex I, III and IV.

Conclusions: Our findings confirmed implication of VPS13D in spastic ataxia and provided further support for mitochondrial defects in patient's skin fibroblasts with VPS13D variants. This report of long-term follow up showed a slowly progressive course of the spastic paraplegia with cerebellar features. Furthermore, the performed functional studies could be used as biomarker helping diagnosis of VPS13D-related neurological disorders when molecular results are uneasy to interpret.
\end{abstract}

Keywords: Spastic ataxia, VPS13D, Whole exome sequencing, Mitochondrial network

\section{Background}

VPS13D is a large ubiquitin-binding protein $(492 \mathrm{kDa})$ which belongs to a family of 4 ubiquitously expressed genes encoding highly conserved proteins in eukaryotic

\footnotetext{
*Correspondence: christelle.durand@u-bordeaux.fr

${ }^{1}$ INSERM U1211, Laboratoire Maladies Rares: Génétique et Métabolisme,

Bordeaux Univ., CHU Bordeaux - Hôpital Pellegrin - Place Amélie Raba

Léon, 33076 Bordeaux Cedex, France

Full list of author information is available at the end of the article
}

cells (VPS13A-D). Recent studies have suggested that Vps13D plays a crucial role in mitochondrial dynamics and is important for mitochondrial integrity [1-3]. Indeed, in Drosophila, Vps13D is required for mitochondrial clearance and is essential in the mitophagy process by regulating mitochondrial fission. Moreover, Vps13D depleted neurons display an incomplete mitophagy with an accumulation of mitophagy intermediates [3]. Depletion of VPS13D in HeLa cells also leads to enlarged and 
rounded mitochondria [2]. Finally, Vps13D is localized at different membrane contact sites and may regulate mitochondria_endoplasmic reticulum contacts, as well as peroxisome biogenesis [4-7]. This localization could be largely determined by competition between specific protein adaptors, all of them binding to VAB (VPS13 adaptor binding domain) [6].

Recently, VPS13D pathogenic variants have been successively reported in patients displaying variable neurological phenotypes generally dominated by movement disorders including chorea, dystonia, tremor, ataxia, spastic paraplegia, spastic ataxia and seizures with highly variable age at onset [8-12]. Progressive spastic ataxia constitutes a genetically heterogeneous group of disorders characterized by simultaneous cerebellar ataxia and limb spasticity, possibly associated with other severe neurological complications [13].

We confirmed herein that spastic ataxia may be related to VPS13D pathogenic variants and we showed abnormal mitochondrial morphology and reduced mitochondrial OXPHOS complexes in skin fibroblasts suggesting altered mitochondrial function.

\section{Narrative}

\section{Case presentation}

The proband (II.4) was a 51-year-old man born from unrelated healthy parents (I.1 and I.2) after an uneventful pregnancy. Familial history was negative (Fig. 1A). He had neither psychomotor delay nor behavioral disorders. His medical history began at age 7 , with acute unilateral
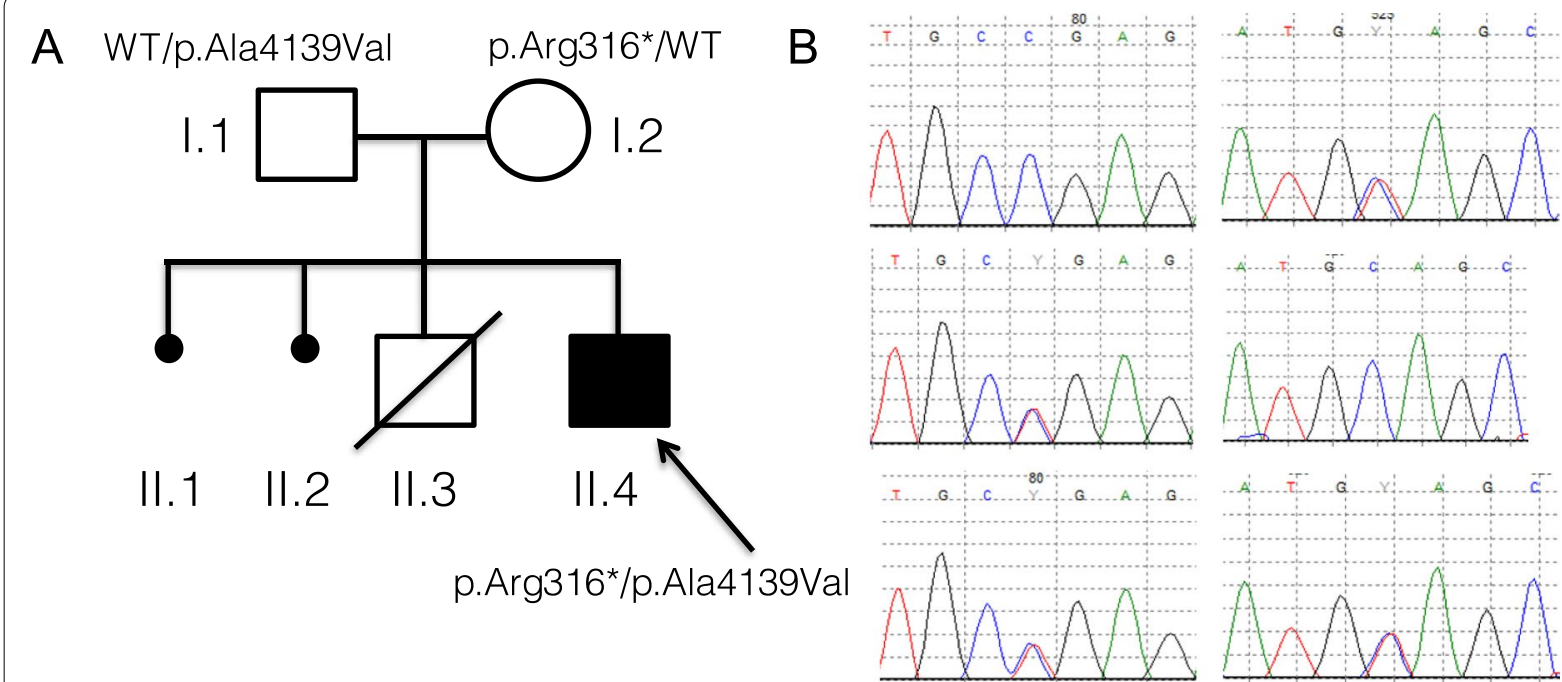

C

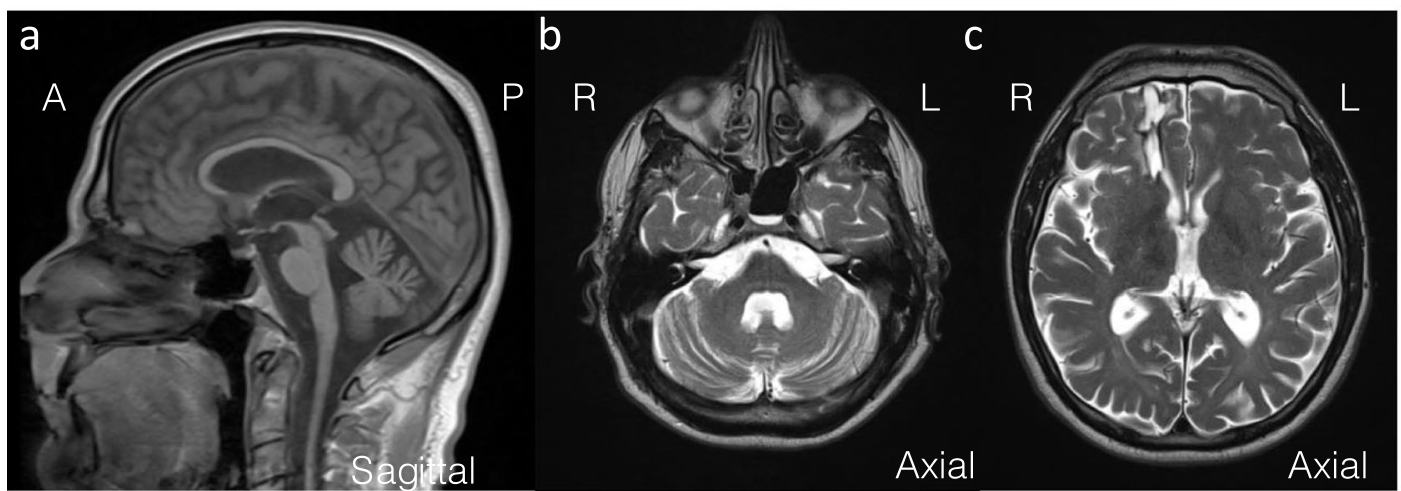

Fig. 1 Family pedigree and brain imaging. A Family Pedigree. B WES identified two variants in VPS13D gene [NM_015378.3] in trans in patient (II.4). The first one is a nonsense variant inherited from his mother (I.2): c.946C > T, p.Arg316*; the second one is a missense variant inherited from his father (I.1): C.12416C > T, p.(Ala4139Val). WT: wild-type. C Brain MRI sections with (C.a) midline sagittal T1 showing vermian atrophy, (C.b) axial T2 showing pontocerebellar and cerebellar atrophy, and (C.C) axial T2, showing frontal angioma therapy after-effects. (A) Anterior; (P) Posterior; (L) Left; (R) Right 
walking difficulties that progressively became bilateral. The walking difficulties were mainly due to spasticity first, with tiptoeing, with no ataxia or weakness. Walking difficulties slowly worsened over time. He walked with a stick at 19 and he's using a wheelchair since age 26 . He presented a generalized onset motor seizure at age 25 that revealed a frontal angioma, treated with radiotherapy. $\mathrm{He}$ presented memory impairment from age 30. Neuropsychological evaluation highlighted moderate difficulties for fronto-parietal efficiency. He complained from dysuria at age 30, and urodynamic testing showed detrusor and external sphincter dyssynergia. Clinical examination found at age 45 a tetraparesis with brisk reflexes, bilateral Hoffmann and Babinski's sign. Spasticity was mostly found on lower limbs with atrophy of proximal muscles. Sensory function was impaired with light touch and vibration deficits, localized preferentially at the lower limbs. He also had a cerebellar kinetostatic ataxia, with truncal hypotonia. He had no extrapyramidal symptoms; cranial nerves examination was normal. No signs of dysmorphia were noted. At age 49, worsening of ataxia and swallowing troubles with episodic false passages were noted. At age 51, he presented focal onset evolving into bilateral convulsive status epilepticus revealing structural epilepsy and requiring chronic use of anti-epileptic medication. Blood analyses revealed normal levels of cholestanol, very long chain fatty acid, normal 25 and 27-hydroxycholesterol plasma level and normal organic and amino acids chromatography. No abnormality was found on urine chromatography of organic acids. At age 51, brain MRI showed atrophic cerebellum mostly on the vermis and an atrophic spinal cord. After-effects of frontal angioma were also present (Fig. 1C).

\section{Whole-exome sequencing (WES), analysis, and interpretation}

We decided to perform trio-based whole-exome sequencing to investigate this patient. Library preparation, exome capture, sequencing and data analysis have been performed by IntegraGen SA (Evry, France), using Twist Bioscience in-solution enrichment methodology (Agilent, Santa Clara, California), followed by pairedend 75 bases massively parallel sequencing on Illumina HiSeq4000 (Illumina, San Diego, California). Wholeexome sequencing for the patient and his parents identified compound heterozygous variants in VPS13D gene [NM_015378.3], with appropriate familial segregation (Fig. 1A, B): c.946C > T, p.Arg316* and c.12416C > T, p.(Ala4139Val). Sanger sequencing using standard protocol was performed to confirm the presence of these variants.

The first variant (rs758368974) was a nonsense variant only present twice at heterozygous state in gnomAD database and considered as pathogenic according to the ACMG guidelines. The second variant was a missense variant absent from gnomAD and predicted as deleterious by PolyPhen-2, LRT and Mutation Taster software. It has a CADD-Phred score of 24.9. These two variants have been submitted in the Clinvar database (SCV001338779.1 and SCV001338853.1).

\section{Mitochondrial studies}

Given the known role of VPS13D on mitochondrial network and integrity, we investigated mitochondrial function and morphology in the patient and in two healthy subjects including one age and gender matched control (ctrl1). Primary fibroblasts were obtained from arm skin biopsies and were cultured under standard conditions [14]. To study mitochondrial morphology, mitochondrial imaging was performed with MitoTracker Green (Fig. 2A). The number of circular mitochondria were quantified using ImageJ software and expressed as a percentage of circular mitochondria per cells as previously described [8]. Patient fibroblasts presented a significant increase of bright and circular mitochondrial objects compared to controls, as previously observed [8], suggesting a structural defect in mitochondria (Fig. 2B). Moreover, by using the Mitochondrial Network Analysis tool from ImageJ platform (MiNA) [15], we showed that summed branch lengths were significantly reduced in patient fibroblasts confirming an altered mitochondrial network (Fig. 2 C). To investigate the effect of VPS13D mutations on the steady-state levels of individual OXPHOS complex subunits in fibroblasts, western blot analysis was performed (Fig. 2) on the fibroblast lysates from patient and ctrl1. Results revealed that in patient cells, there was no apparent effect on the abundance of VDAC1, a mitochondrial outer membrane protein, suggesting no difference in mitochondria content (Fig. 2D). However, a significant decrease in steady-state levels of proteins from complex I, III and IV normalized to VDAC1 was observed in patient fibroblasts (Fig. 2E).

\section{Discussion and conclusions}

VPS13D-related movement disorders have been very recently recognized and to date, only 27 individuals from 18 families have been identified [8-13]. Two main clinical presentations may be distinguished, essentially correlated with age at onset. The first phenotype occurs early after birth, in neonatal or infancy period of life before age 5 years, and leads to evident developmental delay with progressive movement disorders such as chorea, dystonia, spastic paraplegia, ataxia, spastic ataxia and seizures. The disease progressively worsens requiring assistance for walking and wheelchair in early adulthood. Brain MRI abnormalities, suggestive 


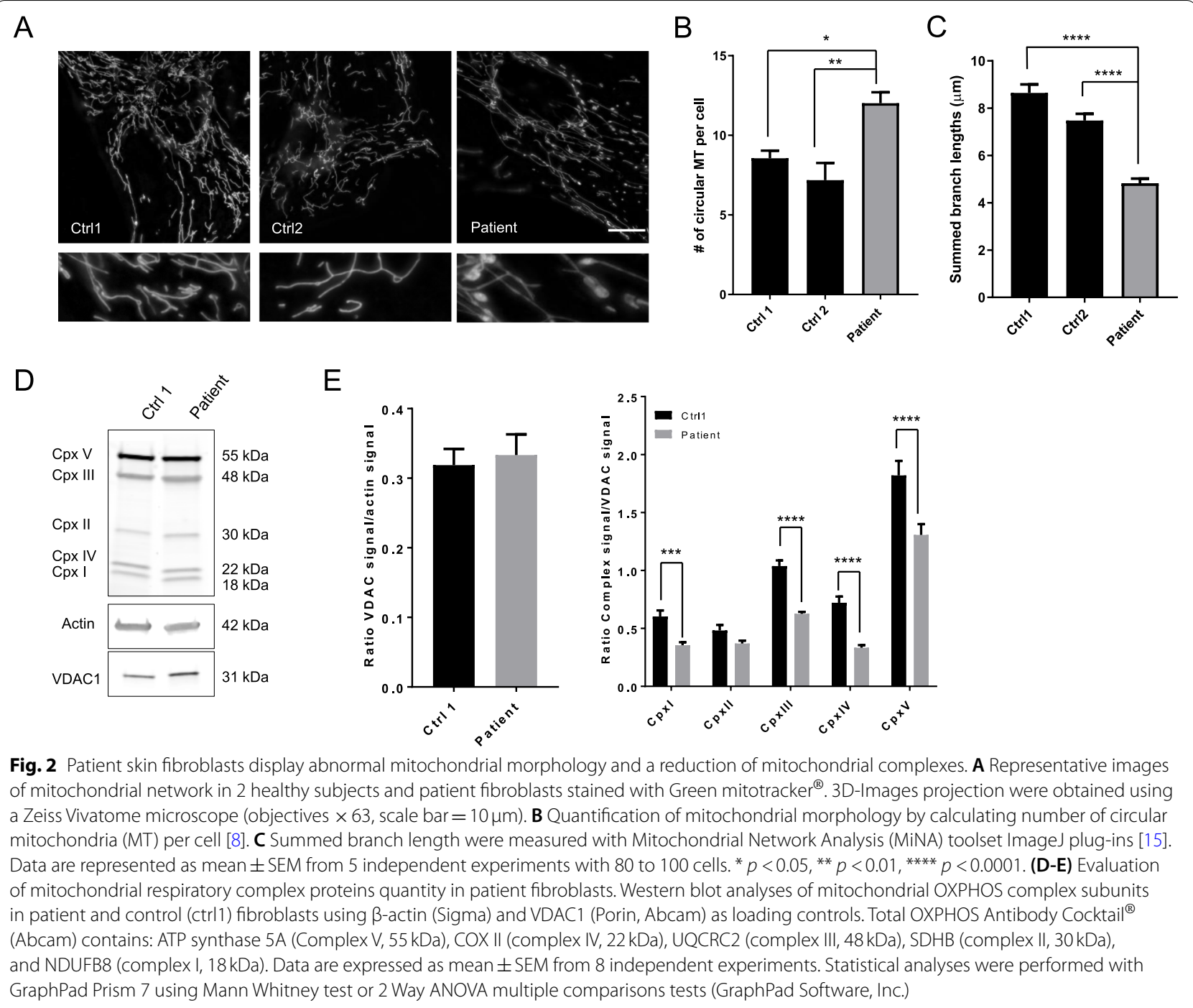

of Leigh syndrome, are frequent. The second phenotype occurs between late childhood and adulthood, after normal early development. The oldest previously reported age at onset was 63 years [10]. The clinical features are then essentially represented by a pure spastic paraplegia or spastic ataxia with tremor and dystonia with progressive course. In this study, we report a novel patient displaying VPS13D-related movement disorders characterized by spastic ataxia which started at age 7 , which corresponds to the second phenotype described above. Cerebellar features became the core motor features around his fifties. In addition, cognitive impairment worsens progressively and is considered one of the most disabling features. However, these cognitive declines may be associated to both sequelae of a treated frontal angioma and potentially undiagnosed focal epilepsy rather than directly caused by the disease.
This patient harbors compound heterozygous nonsense and missense variants as appears to be most commonly found in VPS13D-affected patients [8-12]. Our observations confirm that VPS13D seems intolerant to complete loss of function of both alleles, being likely incompatible with life. Several studies in Drosophila and yeast have shown a major role of Vps13 in two processes that are essential to mitochondrial health: mitochondrial fission and mitophagy $[1-3,7,8]$. Our functional studies in this patient's fibroblasts confirm that mutation in VPS13D induces defects in mitochondrial network and function. Our work support abnormal mitochondrial morphology previously shown in other patients [8]. Moreover, the decrease of some OXPHOS complex subunits observed here argues for additional alteration of mitochondrial function. Further investigations are required to potentially use abnormal mitochondrial morphology as a trait 
biomarker helping diagnosis of VPS13D-related neurological disorders when molecular results are uneasy to interpret and to better understand the functional mitochondrial role of the protein.

\section{Abbreviations}

WES: Whole exome sequencing; MiNA: Mitochondrial Network Analysis toolset ImageJ plug-ins; OXPHOS: Oxidative phosphorylation.

\section{Supplementary Information}

The online version contains supplementary material available at https://doi. org/10.1186/s12883-022-02553-0.

\section{Additional file 1.}

\section{Acknowledgements}

The authors thank the patient and his family for participating in this study, and the Centre of Biology and Pathology of Bordeaux University Hospital for its full support.

\section{Authors' contributions}

CMD performed functional studies and wrote the manuscript. CA, VM, IC contributed to the writing of manuscript. CD and CG performed the clinical follow-up of the patient. AT performed the WES analysis and contributed to the writing of the manuscript. The author(s) read and approved the final manuscript.

\section{Funding}

No specific source of funding was allocated to this study.

\section{Availability of data and materials}

The datasets used and/or analyzed during the current study are available from the corresponding author upon reasonable request.

\section{Declarations}

\section{Ethics approval and consent to participate}

Written informed consent was received from the patient according to the declaration of Helsinki principles of medical research involving human subjects. Experiments were approved by the Comité de Protection des Personnes Bordeaux Outre-Mer III.

\section{Consent for publication}

Written informed consent for publication was received from the patient.

\section{Competing interests}

Dr. Durand received research support from the Association Strumpell-Lorrain (ASL).

Pr Goizet received consulting fees from Overlord, honoraria for lectures from Sanofi Genzyme, Biomarin, Amicus, Biogen, financial support for research activities from Genzyme, Association Strumpell-Lorrain (ASL), Enroll HD, and inscriptions and travels for congresses were funded by Sanofi Genzyme, Amicus and Akcea.

Dr. Trimouille received research support from the Fondation Maladies Rares (FMR).

C Angelini, Dr. Michaud, Dr. Dellci and Dr. Coupry report no disclosures.

\section{Author details}

1'INSERM U1211, Laboratoire Maladies Rares: Génétique et Métabolisme, Bordeaux Univ., CHU Bordeaux - Hôpital Pellegrin - Place Amélie Raba Léon, 33076 Bordeaux Cedex, France. ${ }^{2}$ Centre de Référence Neurogénétique, Service de Génétique Médicale, CHU Bordeaux, Bordeaux, France. ${ }^{3}$ Service de Génétique Médicale, CHU Pellegrin, Bordeaux, France. ${ }^{4}$ Service de Médecine Physique et de Réadaptation Pôle de Neurosciences Cliniques, CHU Pellegrin,
Bordeaux, France. ${ }^{5}$ Univ. Bordeaux, INSERM, BPH, U1219, F-33000, Bordeaux, France. ${ }^{6}$ Service de Pathologie, CHU Pellegrin, Bordeaux, France.

Received: 29 October 2021 Accepted: 9 January 2022

Published online: 12 February 2022

\section{References}

1. Wang Z, Zhang H. Mitophagy: Vps13D couples mitochondrial fission and Autophagic clearance. Curr Biol CB. 2018;28:R66-8.

2. Anding AL, Wang C, Chang T-K, Sliter DA, Powers CM, Hofmann K, et al. Vps13D encodes a ubiquitin-binding protein that is required for the regulation of mitochondrial size and clearance. Curr Biol CB. 2018;28:287-295. e6.

3. Insolera R, Lőrincz P, Wishnie AJ, Juhász G, Collins CA. Mitochondrial fission, integrity and completion of mitophagy require separable functions of Vps13D in Drosophila neurons. PLoS Genet. 2021;17:e1009731.

4. Du Y, Wang J, Xiong J, Fang N, Ji W-K. VPS13D interacts with VCP/p97 and negatively regulates endoplasmic reticulum-mitochondria interactions. Mol Biol Cell. 2021;32:1474-86.

5. Wang J, Fang N, Xiong J, Du Y, Cao Y, Ji W-K. An ESCRT-dependent step in fatty acid transfer from lipid droplets to mitochondria through VPS13DTSG101 interactions. Nat Commun. 2021:12:1252.

6. Dziurdzik SK, Bean BDM, Davey M, Conibear E. A VPS13D spastic ataxia mutation disrupts the conserved adaptor-binding site in yeast Vps13. Hum Mol Genet. 2020;29:635-48.

7. Park J-S, Thorsness MK, Policastro R, McGoldrick LL, Hollingsworth NM, Thorsness $\mathrm{PE}$, et al. Yeast Vps13 promotes mitochondrial function and is localized at membrane contact sites. Mol Biol Cell. 2016;27:2435-49.

8. Seong E, Insolera R, Dulovic M, Kamsteeg E-J, Trinh J, Brüggemann N, et al Mutations in VPS13D lead to a new recessive ataxia with spasticity and mitochondrial defects. Ann Neurol. 2018:83:1075-88.

9. Gauthier J, Meijer IA, Lessel D, Mencacci NE, Krainc D, Hempel M, et al. Recessive mutations in VPS13D cause childhood onset movement disorders. Ann Neurol. 2018;83:1089-95.

10. Koh K, Ishiura H, Shimazaki H, Tsutsumiuchi M, Ichinose $Y$, Nan H, et al. VPS13D-related disorders presenting as a pure and complicated form of hereditary spastic paraplegia. Mol Genet Genomic Med. 2020;8:e1 108.

11. Lee JS, Yoo T, Lee M, Lee Y, Jeon E, Kim SY, et al. Genetic heterogeneity in Leigh syndrome: highlighting treatable and novel genetic causes. Clin Genet. 2020;97:586-94.

12. Petry-Schmelzer JN, Keller N, Karakaya M, Wirth B, Fink GR, Wunderlich G. VPS13D: One Family, Same Mutations, Two Phenotypes. Mov Disord Clin Pract. 2021;8:803-6.

13. Bereznyakova O, Dupré N. Spastic ataxias. Handb Clin Neurol. 2018;155:191-203.

14. Lavie J, Serrat R, Bellance N, Courtand G, Dupuy JW, Tesson C, et al. Mitochondrial morphology and cellular distribution are altered in SPG31 patients and are linked to DRP1 hyperphosphorylation. Hum Mol Genet. 2017:26(4):674-85.

15. Valente AJ, Maddalena LA, Robb EL, Moradi F, Stuart JA. A simple ImageJ macro tool for analyzing mitochondrial network morphology in mammalian cell culture. Acta Histochem. 2017;119:315-26.

\section{Publisher's Note}

Springer Nature remains neutral with regard to jurisdictional claims in published maps and institutional affiliations. 